\title{
The Study of a Control Signal's Phase Shift Influence on the Efficiency of a System for Active Vibration Damping Based on MFC Piezoelectric Transducers
}

\author{
Marek Płaczek ${ }^{1, *}$ \\ ${ }^{1}$ Silesian University of Technology, Department Sciences, Editorial Department, 44-100 Gliwice, \\ Poland
}

\begin{abstract}
Active vibration damping of mechanical systems based on applications of smart materials has a large application potential and is getting more and more popular. In active vibration systems the fast response of actuators to the signals generated by sensors is one of the most important element that decides of the system's efficiency because the idea is to generate force by active elements that will suppress the vibrations detected by the sensors. In this paper results of laboratory tests of a control signal's phase shift influence on the efficiency of a system for active vibration damping based on application of Macro Fiber Composite (MFC) piezoelectric transducers are presented. MFCs are modern piezoelectric composite transducers produced as a thin, elastic films and can be easily installed on the surface of the mechanical subsystem or laminated in composite structures. The impact of the phase shifting between signals generated to power the actuator on the damping efficiency was verified and analysed. It was verified in what phase angle the damping of vibration has the best efficiency and if the shift of the signals causes the linear loss of the system efficiency. It was also verified whether it causes the same effects in both directions of shifting (advance or delay in the phase of the signal supplying the damper relative to the signal generated by the beam's vibration).
\end{abstract}

\section{Active vibration damping using piezoelectric transducers}

The damping of mechanical vibrations is an extremely important issue that is the subject of many works and scientific research [1-6]. Active vibration damping of mechanical systems based on applications of smart materials has a large application potential and is getting more and more popular. The efficiency of such systems is getting better at the same time because of development of sensors and actuators that are used as well as control systems [79]. One of important stages on the path of piezoelectric transducer development was NASA's development of Macro Fiber Composite transducers (MFC) [10]. The use of piezoelectric transducers is an increasingly important and popular element of modern

*Corresponding author: marek.placzek@polsl.pl 
mechatronic systems that combine mechanical, electrical engineering and advanced control systems [11-13]. Piezoelectric transducers enable easy and effective conversion of electrical energy into mechanical energy or in the opposite direction.

Piezoelectric transducers are successfully used in passive vibration reduction systems, in which, together with external, passive electrical circuits, enable the dissipation of mechanical vibration energy. Systems of this type are characterized by simplicity of design and no need to introduce an additional source of energy into the system [14-18]. On the other hand, it is not possible to achieve such vibration damping efficiency as in the case of active systems. In active systems, by using a more complex system consisting of sensors that provide information on current vibration parameters to the control system and actuators controlled by this control system, it is possible to effectively damp vibration. In addition to the greater system complication, these systems also require additional energy to be supplied to the system to power both the control system and the actuators.

Active vibration damping finds a lot of applications in aviation. The example of active vibration control in aviation solutions was the Active Controls eXpert company research, which prepared a system for the damping of a tail of a military aircraft. Currently, they are also used to control aircraft wing flaps. In addition to aviation, piezoelectric systems are also used as vibration dampers for other vehicles, snowboards or baseball bats, for active damping of noise [19]. In paper [20] review of researches in the field of active vibration control and description of the most important methods of their implementation. Nonadaptive and adaptive systems (feedforward, feedback and hybrid control structures, single and multiple channel case) with adaptive algorithms are outlined. Influence and modelling of secondary path is presented. Major applications and directions for further research are indicated. A practical example of vibration damping using piezoelectric transducers is discussed in the article [21]. The article discusses the problem of self-excited vibrations in machining and proposed constructions of a vibration eliminator using a piezo actuator, thanks to which it is possible to reduce vibrations. A control system based on a Gaussian linear-square regulator ensuring high efficiency of the vibration eliminator, which has been confirmed experimentally for various cutting parameters is also presented. The experiment described in this article showed that the use of a piezo actuator allowed to modify the dynamic properties of the workpiece and reduce the level of vibration. The advantages and disadvantages of the presented solution were also indicated. The main ones include the fact that piezo elements provide greater efficiency when working at higher frequencies and are smaller in size than electromagnetic systems. The use of the discussed vibration eliminator does not reduce the machining efficiency, which is a common disadvantage of various types of active and passive methods of vibration elimination during machining. The presented solution also has an advantage over passive solutions because the system retains its efficiency in the event of changes in machining parameters. The only significant disadvantage is the high cost of the actuator and high voltage power amplifier. Also, in paper [22] active vibration control of a plate like smart flexible structure is presented. This plate is equipped with several thin piezoelectric patches. Some of them are used as sensors and the others as actuators. They are optimally positioned and not collocated. The main goal of control is to reduce the most energetic vibrating modes.

In active vibration systems the fast response of actuators to the signals generated by sensors is one of the most important element that decides of the system's efficiency because the idea is to generate force by active elements that will suppress the vibrations detected by the sensors. In this paper results and their analysis of laboratory tests of a control signal's phase shift influence on the efficiency of a system for active vibration damping based on application of Macro Fiber Composite (MFC) piezoelectric transducers are presented. MFCs are modern piezoelectric composite transducers produced as a thin, elastic films and can be easily installed on the surface of the mechanical subsystem or laminated in 
composite structures. They can be use both as sensors or as actuators based on direct and reverse piezoelectric effects. Therefore, the potential of their applications in variety types of mechatronic systems is very high, including systems for active vibration damping. Presented laboratory tests were conducted using a mechanical subsystem in the form of cantilever beam excited with the first natural frequency of vibration by the MFC actuator. The impact of the phase shifting between signals generated to power the actuator and damper on the damping efficiency was verified and analysed. It was verified in what phase angle the damping of vibration has the best efficiency and if the shift of the signals causes the linear loss of the system efficiency. It was also verified whether it causes the same effects in both directions of shifting (advance or delay in the phase of the signal supplying the damper relative to the signal generating the beam's vibration).

\section{Laboratory stand for testing the influence of phase shift angle of signals in the system of active damping of beam's vibrations}

In order to verify the impact of the phase shift angle on the efficiency of the active vibration damping system based on the use of MFC piezoelectric transducers, a laboratory stand was built, which consists of:

- a clamp with the possibility of mounting the tested piece,

- a mechanical subsystem (a cantilever beam made of an aluminium alloy),

- a generator of signals with required frequency WW5064 by Tabor Electronics,

- two high voltage amplifiers HVA $1500 / 50$ by Smart Material,

- an oscilloscope RIGOL DS1064B

- MFC M8514-P1 and M8507-P1 piezoelectric transducers.

The created stand for laboratory tests is shown in Fig. 1.
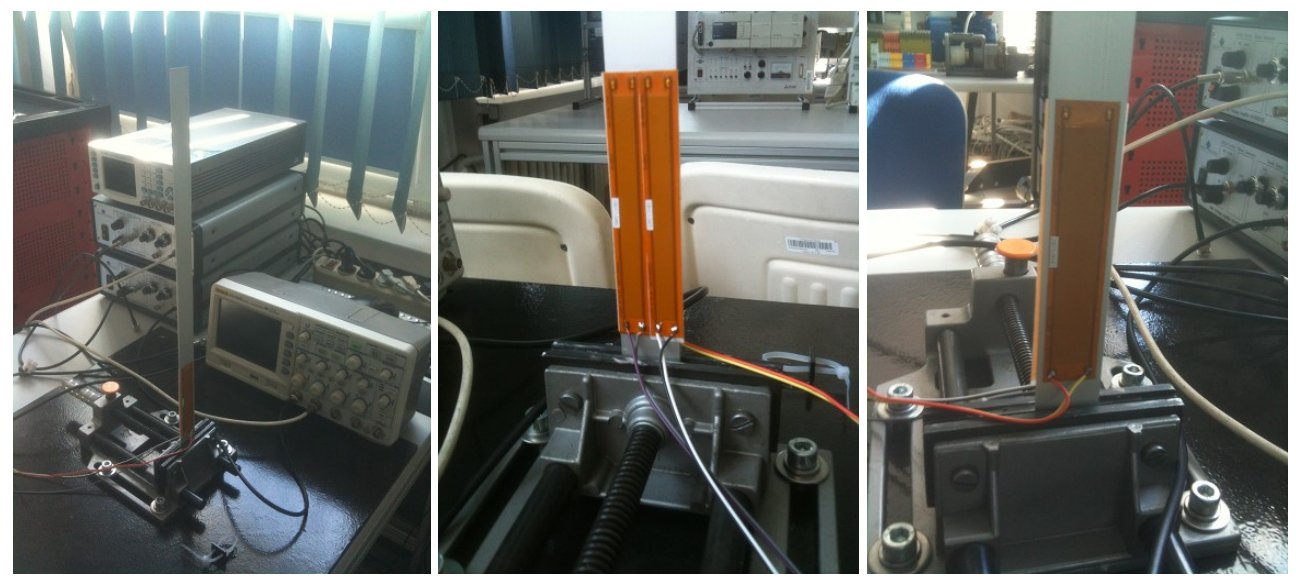

Fig. 1. The created laboratory stand $(a)$, piezoelectric MFC actuator and damper $(b)$ and the MFC transducer used as sensor $(c)$.

Presented laboratory tests were conducted using a mechanical subsystem in the form of cantilever beam excited with the first natural frequency of vibration by the MFC actuator. Three MFC piezoelectric transducers were glued on the surface of the tested beam. The first one was used to generate vibration of the beam, while the second one was used as a damper. The third MFC was used to measure the beam's vibrations and to analyse of the damping efficiency. The vibration generator and damper were powered using laboratory signal generator and high voltage amplifiers. The signal generated to drive the vibration 
damper was phase shifted relative to the actuator supply signal and efficiency of the vibration damping was measured.

The system was excited with a frequency equal to the first natural frequency, which in the case of the considered beam was $8 \mathrm{~Hz}$. Such action was aimed at obtaining maximum values of the amplitude of vibration of the mechanical system. The MFC M8514-P1 transducer was used to indirectly measure the amplitude of vibration of the mechanical subsystem. The value of voltage generated by this transducer depends on its deformation, resulting from the beam deflection and the frequency of deformations, which was constant during all tests. At a constant value of the frequency of excitation of beam vibrations, the value of voltage generated by the MFC sensor was therefore influenced only by the amplitude of the system vibrations.

\section{Measurements results}

The tests were carried out at five different values of the phase shift angle between the signal supplying the transducer exciting vibrations and the signal supplying the piezoelectric damper. Values from $0,8 \pi$ to $1,2 \pi$ with a gradient of $0,1 \pi$ were assumed. This corresponds to the situation in which the signal supplying the active vibration damper in phase is faster than the signal supplying the vibration generator (when $\varphi<\pi)$, is in the opposite phase $(\varphi=$ $\pi)$ or is delayed in phase relative to the vibration excitation signal $(\varphi>\pi)$. The tests were carried out in such a way that vibrations of the mechanical system were forced, and then after some time the power supply of the vibration damper was turned on. After stabilization of the damped oscillation amplitude, the damper was turned off while the actuator of vibrations was still powered. The tests were repeated five times for each phase shift angle value, simultaneously the signal generated by the MFC transducer being the measuring element was recorded.

Fig. 2 shows the waveforms of the electric voltage as a function of time. It is a signal generated by the MFC transducer, which is a measurement element of the effectiveness of the active vibration damping system. The vertical lines in black indicate the moment of activation of the system of active vibration damping, while the vertical lines in red indicate the moment of system deactivation, while the vibrations of the mechanical system were still excited. The waveforms are presented at different values of the phase shift angle $\varphi$ of the signal, which fed the transducer acting as a vibration damper in relation to the signal supplying the actuator which excite system vibration.

Table 1 summarizes the values of the average maximum amplitude of the signal generated by the measuring transducer after stabilization of the system vibration amplitude during the operation of the active vibration damping system.

Table 1. Average value of the voltage amplitude generated by the measuring transducer.

\begin{tabular}{|c|c|}
\hline Signals phase shift angle [rad] & $\mathbf{V}_{\max }[\boldsymbol{V}]$ \\
\hline $0,8 \pi$ & 2,42 \\
\hline $0,9 \pi$ & 1,31 \\
\hline $1,0 \pi$ & 0,35 \\
\hline $1,1 \pi$ & 0,62 \\
\hline $1,2 \pi$ & 1,62 \\
\hline
\end{tabular}




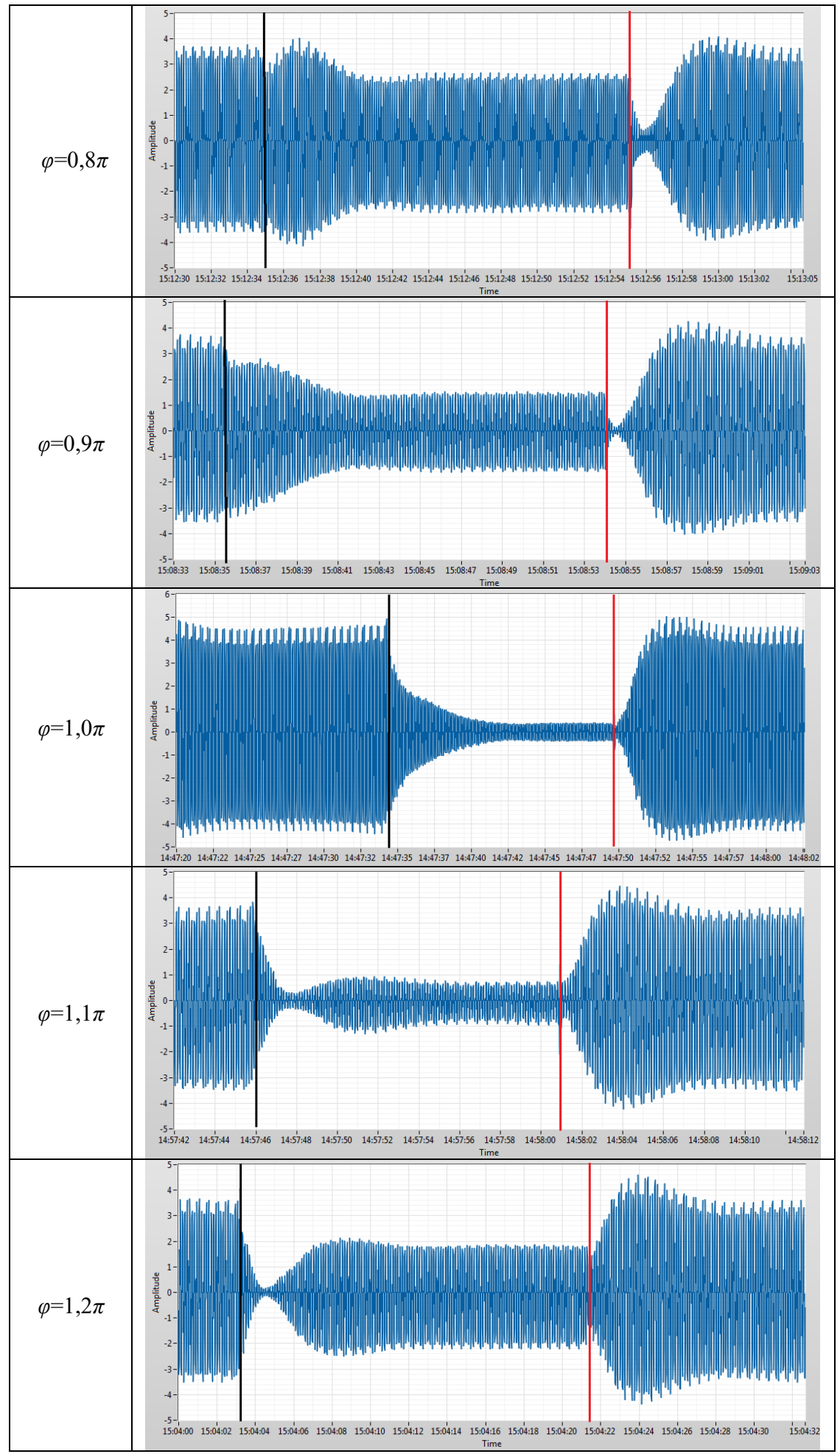

Fig. 2. Time waveforms of the voltage signal generated by the MFC transducer acting as a measuring element with different angles of phase shift of the signals. 


\section{Conclusions}

Laboratory measurements of the effectiveness of the active vibration damping system at variable phase shift angle values between the excitation signal of the mechanical system and the signal supplying the piezoelectric damper have shown its high significance for the efficiency of vibration damping of the system. To achieve maximum vibration damping efficiency, these signals must be in the opposite phase (shifted by $1.0 \pi$ radians). Any deviation from this value causes a decrease in the effectiveness of the system. It should be emphasized that in the event of a delay in the phase of the signal supplying the piezoelectric damper relative to the excitation signal $(\varphi>\pi)$, the decrease in system performance is smaller than in the case when the signal supplying the piezoelectric damper is faster in the phase than the beam deformation resulting from its excitation $(\varphi<\pi)$. It should also be noted that in the event that $\varphi>\pi$ after switching on active vibration reduction, there is a transition period in which the vibration damping efficiency is very high, followed by a decrease in efficiency and stabilization of the vibration amplitude at a certain level. If $\varphi<\pi$, this phenomenon occurs when the active vibration reduction system is turned off. When designing active vibration reduction systems based on the use of piezoelectric transducers, these phenomena should be considered and strive to develop fast control algorithms in which it will be possible to achieve maximum efficiency and effectiveness of vibration damping.

\section{References}

1. K. Białas, Solid State Phenomena 164 (2010)

2. K. Białas, J. of Vibroengineering 14 (2012)

3. A. Dymarek, T. Dzitkowski, J. of Materials Processing Technology 164-165 (2005)

4. A. Dymarek, T. Dzitkowski, J of Vibroengineering 15, 3 (2013)

5. A. Wróbel, J. of Vibroengineering 14, 2 (2012)

6. S. Zolkiewski, Int. J. of Bifurcation and Chaos 21, 10 (2011)

7. T. Bartel, S. Herold, D. Mayer, T. Melz, Proceedings of the $6^{\text {th }}$ ECCOMAS Conference on Smart Structures and Materials (2013)

8. S. Herold, D. Mayer, Actuators 5, 7 (2016)

9. L. Sui, X. X. Shi, Physics Procedia 25 (2012)

10. M. Płaczek, G. Kokot, Sensors 19, 2 (2019)

11. W. Banaś, A. Sękala, K. Foit, et al., IOP Conf. Series: Mat. Sc. and Eng. 95 (2015)

12. A. Gwiazda, K. Herbuś, G. Kost, P. Ociepka, Solid State Phenomena 220-221 (2015)

13. A. Gwiazda, A. Sękala, Z. Monica, W. Banaś, Adv. Mat. Res. B (2014)

14. A. Buchacz, M. Płaczek, A. Wróbel, Eksploatacja i niezawodnosc - Maintenance and Reliability 16, 2 (2014)

15. M. Płaczek, Advanced Material Research 1036 (2014)

16. A. Buchacz, M. Płaczek, Solid State Phenomena 147-149 (2009)

17. K. Marakakis, G. Tairidis, P. Koutsianitis, G. Stavroulakis, Front. Built Environ. 5 (2019)

18. Z. Skup, J. of Theoretical and Applied Mechanics 44, 1 (2003)

19. S. Yoshikawa, A. Bogue, B. Degon, Proceedings of the $11^{\text {th }}$ IEEE International Symposium on Applications of Ferroelectrics (1998)

20. D. Milijkovic, MIPRO 2009 Conference Proceedings (2009)

21. A. Parua, K. Marchelek, S. Domek, M. Hoffmann, Modelowanie Inzynierskie Modelling in Engineering 39 (2010)

22. S. Tliba, H. Abou-Kandil, C. Prieur, IFAC Elsevier Publications, $16^{\text {th }}$ Triennial World Congres, Prague, Czech Republic (2005) 